Eiter mit intrazellulär gelegenen Gonokokken. Später nur Micrococcus ureae.

Von einer radikalen Exstirpation des Ganges wurde abgesehen und die Ausspritzung desselben mittels einer Zahnspritze mit $1 / \%$ Lapislösung empfohlen.

Richard Fischel (Bad Hall).

\title{
Japanische Zeitschrift für Dermatologie und Urologie.
}

Vol. XIII. Fasc. 1. Januar 1913.

Maki. Histologische Untersuchung des Lupus erythematoses disseminatus.

Ijiri, Über Tiosinaminvergiftung.

Ijiri stellt aus der Literatur 21 Fälle von Tiosinaminvergiftung und 5 Fälle von Tiosinaminexanthemen zusammen und fügt einen Fall eigener Beobachtung hinzu, der eine 33jährige Frau betraf, die wegen Narbenkontrakturen nach Hautgummen behandelt wurde. Sie erhielt 0.2 bis $1 \mathrm{ccm}$ der $15 \%$ igen alkoholischen und der 10\% igen glyzerin-wäßrigen Lösung. Nach der dritten Injektion Herzklopfen und leichte Temperatursteigerung. Fünf Tage später (trotzdem das Mittel inzwischen ausgesetzt worden war) Ohnmacht, Herzklopfen, Dyspnoe, Üblichkeit, Erbrechen. Zwanzig Tage nach ihrer Wiederherstellung wurden durch eine Injektion von $1 \mathrm{ccm}$ der 10\% gelöst.

Walter Pick (Wien).

\section{Geschlechts-Krankheiten.}

\section{Syphilis. Allgemeiner Teil.}

Melville, Stanley. Zur Anzeigepflicht venerischer Krankheiten. The Practitioner. Juli. 1912.

Die Ausfïhrungen M. führen ihn zu folgenden Forderungen: 1. Staatliche Maßregeln zur Verhütung und Unterdrückung der Geschlechtskrankheiten. 2. Unterdrückung der jugendlichen Prostitution. 3. Bestrafung der wissentlichen Übertragung von Geschlechtskrankheiten. 4. Einführung der Anzeigepflicht für Geschlechtskrankheiten und Vorsorge zur Behandlung der Erkrankten.

Wilhelm Balban (Wien). 